\title{
Nonpulmonary risk factors of acute respiratory distress syndrome in patients with septic bacteraemia
}

Hyunseung Nam ${ }^{1}$, Seung Hun Jang ${ }^{2}$, Yong Il Hwang ${ }^{2}$, Joo-Hee Kim², Ji Young Park², and Sunghoon Park²

Departments of ${ }^{1}$ Internal Medicine and ${ }^{2}$ Pulmonary, Allergy and Critical Care Medicine, Hallym University Sacred Heart Hospital, Anyang, Korea
Background/Aims: The relationship between nonpulmonary organ failure and the development of acute respiratory distress syndrome (ARDS) in patients with sepsis has not been well studied.

Methods: We retrospectively reviewed the medical records of patients with septic bacteremia admitted to the medical intensive care unit (ICU) of a tertiary academic hospital between January 2013 and December 2016.

Results: The study enrolled 125 patients of median age 73.0 years. Urinary $(n=47)$, hepatobiliary $(n=30)$, and pulmonary infections $(n=28)$ were the most common causes of sepsis; the incidence of ARDS was 17.6\%. The total number of nonpulmonary organ failures at the time of ICU admission was higher in patients with ARDS than in those without $(p=0.011)$, and the cardiovascular, central nervous system (CNS), and coagulation scores were significantly higher in ARDS patients. On multivariate analysis, apart from pneumonia sepsis, the CNS (odds ratio [OR], 1.917; 95\% confidence interval [CI], 1.097 to 3.348) and coagulation scores (OR, 2.669; $95 \%$ CI, 1.438 to 4.954) were significantly associated with ARDS development. The 28-day and in-hospital mortality rates were higher in those with ARDS than in those without (63.6 vs. $8.7 \%, p<0.001 ; 72.7 \%$ vs. $11.7 \%, p<0.001$ ), and ARDS development was found to be an independent risk factor for 28 -day mortality.

Conclusions: Apart from pneumonia, CNS dysfunction and coagulopathy were significantly associated with ARDS development, which was an independent risk factor for 28-day mortality.

Keywords: Respiratory distress syndrome, adult; Bacteremia; Organ failure; Sepsis

\section{INTRODUCTION}

Sepsis remains a major public health problem, accounting for $5.2 \%$ of the total United States hospital costs in 2011, and is the primary cause of death from infection [1]. The development of acute respiratory distress syndrome (ARDS) is not uncommon among patients with sepsis, triggered by a direct lung injury caused by pneu- monia or an indirect lung injury attributable to infection of a distant organ (e.g., acute pancreatitis).

Less is known about the relationship between nonpulmonary organ failure and ARDS development. One multicenter observational study found that pneumonia sepsis was associated with ARDS development [2]. In another observational prospective study, $4.6 \%$ of patients with extrapulmonary conditions developed 
ARDS. The likelihood of developing ARDS (or acute lung injury) was $35.6 \%$ in patients with shock and $1.4 \%$ in those with nonpulmonary sepsis without shock [3]. However, because the lungs are one of the targets of an overwhelming acute systemic inflammatory response, the recognition of nonpulmonary organ failure as a risk factor for developing ARDS seems to be important [4].

Multiorgan dysfunction in patients with sepsis is associated with increased in-hospital mortality. However, the data on the association between ARDS development and in-hospital mortality in patients with sepsis are conflicting. Two studies reported that, after adjusting for confounding factors, ARDS development after sepsis onset was not associated with an increased risk of mortality $[5,6]$, while an epidemiological study found the opposite [7]. Therefore, we explored whether ARDS is a risk factor for hospital death in patients with sepsis.

In this study, we evaluated nonpulmonary organ failure associated with the risk of ARDS development and the impact of ARDS on mortality in patients with septic bacteremia.

\section{METHODS}

\section{Study population}

This retrospective study was performed in a tertiary academic hospital (830 beds) from January 1, 2013 to December 31, 2016. Adult patients (aged $\geq 18$ years) with septic bacteremia admitted to the medical intensive care unit (ICU) were screened initially. Bacteremia was diagnosed when at least two blood cultures were positive. The exclusion criteria were (1) a positive blood test result believed to be caused by contamination (i.e., only one positive culture); (2) liver cirrhosis ( $\geq$ Child-Pugh B grade); (3) an uncontrolled hematological malignancy or a solid cancer; (4) use of steroids or immunosuppressants; (5) cardiopulmonary resuscitation upon ICU admission; and (6) a do-not-resuscitate status. However, cancer patients were eligible if they had been in complete remission for $>6$ months, and patients who were taking low-dose steroids (i.e., $\leq 10 \mathrm{mg}$ /day prednisolone or the equivalent) were also eligible. We used the Sepsis-3 definition to diagnose sepsis $[8,9]$ and followed the treatment recommendations of the Surviving Sepsis Campaign $[10,11]$. We used the Berlin definition of
ARDS [12]. We measured ARDS incidence during the first week of sepsis and classified ARDS severity according to the ratio of the arterial oxygen tension to the inspired oxygen fraction $\left(\mathrm{PaO}_{2} / \mathrm{FiO}_{2}\right)$ : mild $\left(200<\mathrm{PaO}_{2} /\right.$ $\left.\mathrm{FiO}_{2} \leq 300 \mathrm{mmHg}\right)$, moderate $\left(100<\mathrm{PaO}_{2} / \mathrm{FiO}_{2} \leq 200\right.$ $\mathrm{mmHg})$, and severe ARDS $\left(\mathrm{PaO}_{2} / \mathrm{FiO}_{2} \leq 100 \mathrm{mmHg}\right)$.

The study was approved by the Hallym University Institutional Review Board (approval no. 2017-Io49). The need for informed consent was waived because of the retrospective nature of the work.

\section{Data collection and outcomes}

We retrieved demographic characteristics and the following data by retrospective review of medical records: body mass index, the cause of sepsis, the organisms identified (and whether they were Gram-positive or -negative), sequential organ failure assessment (SOFA) scores and scores on the individual components thereof, and simplified acute physiology score II (SAPS II) at ICU admission. Laboratory data (i.e., the levels of lactate, C-reactive protein, and brain natriuretic peptide [BNP]), the appropriateness of empirical antibiotics, and bacterial multidrug resistance were also investigated. We used the six individual components of the SOFA to assess organ failure, which was performed before ARDS developed; organ failure was considered present when any individual score was $\geq 2$.

The primary outcomes were the associations between nonpulmonary organ failure and ARDS development; we also sought independent factors predicting ARDS development. The secondary outcome was the effect of ARDS on 28-day mortality.

\section{Statistical analysis}

All categorical variables are presented as numbers with percentages, and all continuous variables as medians with interquartile ranges (IQRs), while survival times are presented as means and standard deviation. The Mann-Whitney $U$ test was used to compare continuous variables, and the chi-square or Fisher's exact test to compare categorical variables. Logistic regression analysis was performed using covariates that were significant $(p<0.05)$ on univariate analysis to identify independent risk factors for ARDS development; we employed a backward stepwise selection method based on the likelihood ratio. Kaplan-Meier survival curves 


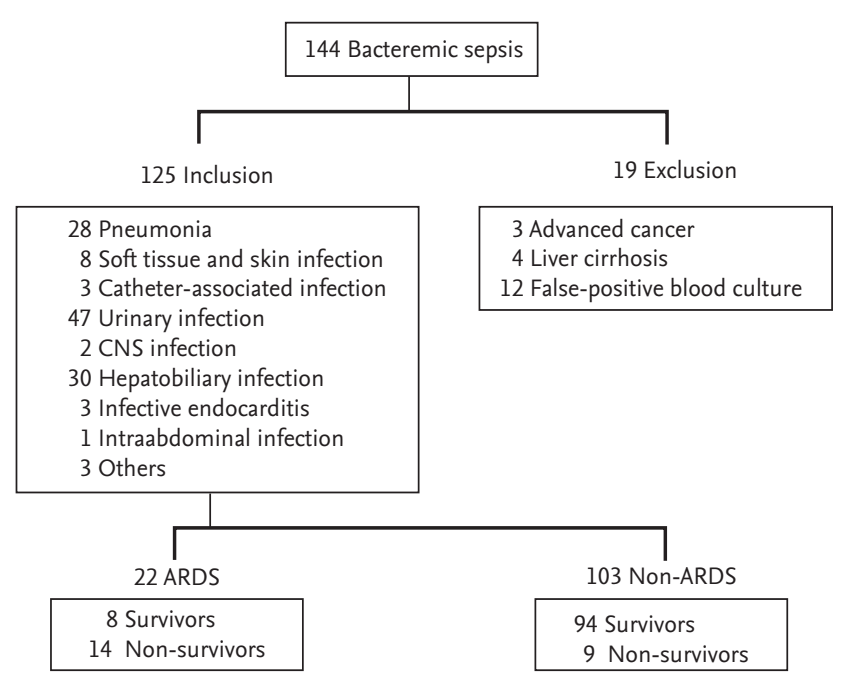

Figure 1. Flow chart of patient enrolment into this study. CNS, central nervous system; ARDS, acute respiratory distress syndrome. with log-rank tests and Cox proportional hazard models based on a multivariate approach were also used. IBM SPSS for Windows version 22.01 (IBM Co., Armonk, NY, USA) was used for all statistical analyses.

\section{RESULTS}

\section{Baseline characteristics}

During the study period, 144 patients with septic bacteremia were screened initially; of these, 125 were enrolled (Fig. 1). The median patient age was 73.0 years (IQR, 59.0 to 80.0 ), and 67 were male (Table 1). Sepsis and septic shock accounted for $51.2 \%$ and $48.8 \%$ of all patients, respectively; urinary tract infection $(n=47)$ was the most common cause of sepsis, followed by hepatobiliary infection ( $\mathrm{n}=30)$. Methicillin-resistant Staphylococcus aureus was the most common Gram-positive pathogen

Table 1. Comparisons of baseline characteristics between ARDS and non-ARDS patients

\begin{tabular}{|c|c|c|c|c|}
\hline Characteristic & All patients $(n=125)$ & $\operatorname{ARDS}(n=22)$ & Non-ARDS $(\mathrm{n}=103)$ & $p$ value \\
\hline Age, yr & $73(59.0-80.0)$ & $65.0(58.0-78.3)$ & $73(60-81)$ & 0.176 \\
\hline Sex, male/female & $67 / 58$ & $12 / 10$ & $55 / 48$ & 0.922 \\
\hline \multicolumn{5}{|l|}{ Comorbid illness } \\
\hline Diabetes & $54(43.2)$ & $10(45 \cdot 5)$ & $44(42.7)$ & 0.814 \\
\hline Hypertension & $62(49.6)$ & $9(40.9)$ & $53(51.5)$ & 0.369 \\
\hline COPD & $3(2.4)$ & 0 & $3(2.9)$ & 1.000 \\
\hline CKD & $21(16.8)$ & $2(9.1)$ & $19(28.4)$ & 0.363 \\
\hline Heart diseases & $18(14.4)$ & $4(18.2)$ & $14(13.6)$ & 0.587 \\
\hline Cancer & $10(8.0)$ & $2(9.1)$ & $8(7.8)$ & 0.689 \\
\hline Pneumonia vs. nonpneumonia & $28 / 97$ & $10 / 12$ & $18 / 85$ & 0.006 \\
\hline Sepsis/septic shock & $64 / 61$ & $11 / 11$ & $53 / 50$ & 0.901 \\
\hline $\mathrm{PaO}_{2} / \mathrm{FiO}_{2}$ & $250.0(180.0-303.5)$ & $165.0(87.5-277.5)$ & $254(210-320)$ & 0.002 \\
\hline Lactate, $\mathrm{mmol} / \mathrm{L}$ & $3.6(2.4-5.8)$ & $4 \cdot 3(2.8-9.4)$ & $3.2(2.3-5.6)$ & 0.123 \\
\hline $\mathrm{BNP}, \mathrm{pg} / \mathrm{mL}$ & $297.4(138.5-973.4)$ & $772.1(115.1-1,389.0)$ & $287.2(138.7-927.5)$ & 0.169 \\
\hline Blood urea nitrogen & $31.1(20.4-55.0)$ & $43.4(36.7-64.0)$ & $29.8(19.2-53.7)$ & 0.033 \\
\hline C-reactive protein & $172.9(127.4-231.5)$ & $195.9(174.3-298.6)$ & $167.2(113.0-218.0)$ & 0.011 \\
\hline SOFA & $7.0(5.5-9.0)$ & $10.5(7.0-15 \cdot 3)$ & $7.0(5.0-9.0)$ & $<0.001$ \\
\hline SAPS II & $44.0(35.0-56.0)$ & $58.0(39.8-73.8)$ & $41.0(35.0-53.0)$ & 0.001 \\
\hline
\end{tabular}

Values are presented as median (interquartile range) or number (\%).

ARDS, acute respiratory distress syndrome; COPD, chronic obstructive pulmonary disease; CKD, chronic kidney disease; $\mathrm{PaO}_{2} / \mathrm{FiO}_{2}$, ratio of arterial oxygen tension to inspired oxygen fraction; BNP, brain natriuretic peptide; SOFA, sequential organ failure assessment; SAPS, simplified acute physiology score. 
Table 2. Isolated organisms among enrolled patients

\begin{tabular}{lc}
\hline Organisms identified & No. $(\%)$ \\
\hline Gram-positive organisms & $7(5.6)$ \\
\hline Streptococcus pneumoniae & $1(0.8)$ \\
\hline Streptococcus viridans & $17(13.6)$ \\
Methicillin-sensitive Staphylococcus aureus & $3(2.4)$ \\
Methicillin-resistant Staphylococcus aureus & $3(2.4)$ \\
Other organisms ${ }^{\text {a }}$ & \\
Gram-negative organisms & $52(41.6)$ \\
\hline Escherichia coli & $26(20.8)$ \\
Klebsiella pneumoniae & $3(2.4)$ \\
Pseudomonas aeruginosa & $3(2.4)$ \\
Proteus mirabilis & $3(2.4)$ \\
Citrobacter freundi & $3(2.4)$ \\
\hline Acinetobacter baumannii & $4(3.2)$ \\
\hline Other organisms & \\
\hline
\end{tabular}

${ }^{\mathrm{a}}$ Enterococcus faecalis $(\mathrm{n}=1)$, Staphylococcus hominis $(\mathrm{n}=1)$, and Staphylococcus epidermidis $(\mathrm{n}=1)$.

${ }^{\mathrm{b}}$ Enterobacter aerogenes $(\mathrm{n}=1)$, Serratia marcescens $(\mathrm{n}=1)$, Enterobacter cloacae $(\mathrm{n}=1)$, and Edwardsiella tarda $(\mathrm{n}=1)$.

(17/31) and Escherichia coli the most common Gram-negative bacterium (52/94) (Table 2). Forty-three patients (34.4\%) were infected by multidrug-resistant pathogens, and 11 received inappropriate empirical antibiotics (Table 3). The overall incidence of ARDS was 17.6\% (22/125); those with pneumonia and skin and soft tissue infections had ARDS incidences of $35.7 \%(10 / 28)$ and $37.5 \%$ (3/8), respectively (Fig. 2). The median interval between ICU admission and ARDS development was 1.0 days (range, 0.5 to 3.0), and the number of patients with mild, moderate, and severe ARDS was four (18.2\%), five (22.7\%), and 13 (59.1\%), respectively. Regarding hospital outcomes, both 28 -day and in-hospital mortality rates were higher in patients with ARDS than in those without ARDS (63.6\% vs. 8.7\%, $p<0.001 ; 72.7 \%$ vs. $11.7 \%, p<$ 0.001) (Table 3).

\section{Organ failure and ARDS development}

Patients who developed ARDS had higher SAPS II and SOFA scores at the time of ICU admission compared with those who did not develop ARDS (Table 1). Of the six components of the SOFA score, the cardiovascular, central nervous system (CNS), respiratory, and coagulation scores were significantly higher in ARDS patients
Table 3. Treatments and outcomes between ARDS and non-ARDS patients

\begin{tabular}{lcccc}
\hline Variable & $\begin{array}{c}\text { All } \\
\text { patients } \\
(\mathrm{n}=125)\end{array}$ & $\begin{array}{c}\text { ARDS } \\
(\mathrm{n}=22)\end{array}$ & $\begin{array}{c}\text { Non- } \\
\text { ARDS } \\
(\mathrm{n}=103)\end{array}$ & p value \\
\hline MDR pathogens & $43(34.3)$ & $5(22.7)$ & $38(36.9)$ & 0.204 \\
$\begin{array}{l}\text { Gram(+)/(-) } \\
\text { pathogens }\end{array}$ & $31 / 94$ & $10 / 12$ & $21 / 82$ & 0.013 \\
$\begin{array}{l}\text { Inappropriate } \\
\text { antibiotics }\end{array}$ & $11(8.9)$ & $2(9.1)$ & $9(8.8)$ & 1.000 \\
$\begin{array}{l}\text { Mechanical } \\
\text { ventilation }\end{array}$ & $35(28.5)$ & $15(68.2)$ & $20(19.4)$ & $<0.001$ \\
$\begin{array}{l}\text { Vaosopressors } \\
\begin{array}{l}\text { ICU mortality } \\
\text { 28-Day }\end{array}\end{array}$ & $24(64.8)$ & $16(72.7)$ & $65(63.1)$ & 0.467 \\
\hline $\begin{array}{l}\text { mortality } \\
\begin{array}{l}\text { Hospital } \\
\text { mortality }\end{array}\end{array}$ & $23(18.4)$ & $14(63.6)$ & $9(8.7)$ & $<0.001$ \\
\hline
\end{tabular}

Values are presented as number (\%).

ARDS, acute respiratory distress syndrome; MDR, multidrug resistance; ICU, intensive care unit.

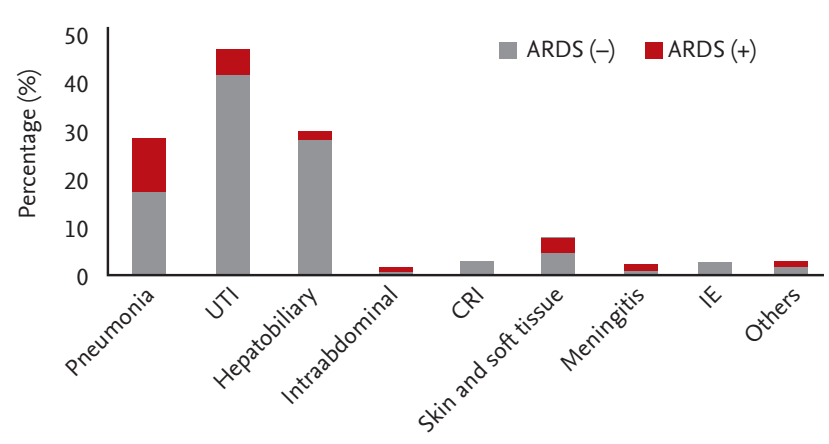

Figure 2. Acute respiratory distress syndrome (ARDS) frequencies according to the origin of sepsis. UTI, urinary tract infection; CRI, catheter-related infection; IE, infectious endocarditis.

(Fig. 3A), and the incidence of ARDS increased with the number of nonpulmonary organ failures (Fig. 4). In addition, the cardiovascular, CNS, respiratory, renal, and coagulation scores were significantly higher in non-survivors than in survivors (Fig. $3 \mathrm{~B}$ ).

\section{Risk factors for ARDS development}

Univariate analyses indicated that a total of 12 variables (eight baseline variables and four organ failure scores) differed significantly between patients who did and 

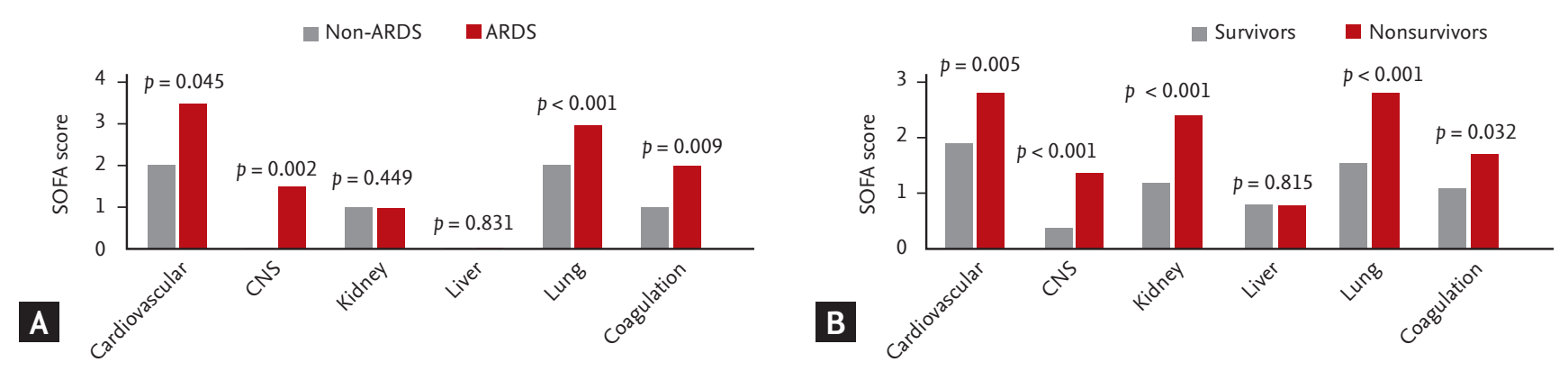

Figure 3. Individual organ failure scores (A) of patients with and those without acute respiratory distress syndrome (ARDS) and (B) of 28-day survivors and non-survivors. SOFA, sequential organ failure assessment; CNS, central nervous system.

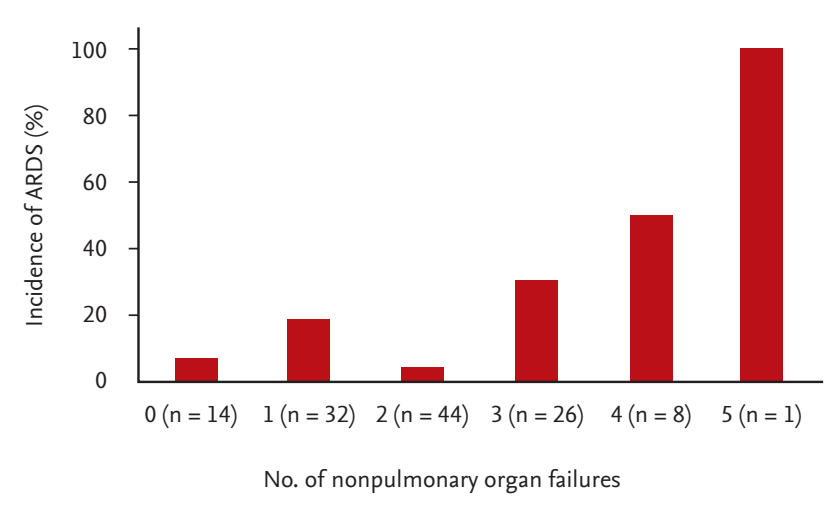

Figure 4. Acute respiratory distress syndrome (ARDS) incidence according to the number of nonpulmonary organ failures. The incidence of ARDS increased in patients with septic bacteremia and with the number of nonpulmonary organ failures.

those who did not develop ARDS (Table 1 and Fig. 3A). After considering overlaps in significance, we selected eight variables (pneumonia sepsis, Gram-negative infection, blood urea nitrogen level, C-reactive protein level, three organ failure scores [cardiovascular, CNS, and coagulation scores], and the number of nonpulmonary organ failures), as well as age and gender, to enter into the multivariate model. Seven variables remained in the final model (Table 4); among them, pneumonia sepsis (odds ratio [OR], 4.590), the coagulation score (OR, 2.669), the CNS score (OR, 1.917), and the C-reactive protein level (OR, 1.007) were independently associated with ARDS development.

\section{ARDS development and 28-day mortality}

In the univariate analyses, 16 variables (11 baseline variables and five organ failure scores) differed significantly between survivors and nonsurvivors (Table 5 and Fig. 3B). After considering overlaps in significance, we initially selected 11 variables (heart disease, Gram-negative infection, BNP, blood urea nitrogen, lactate, ARDS development, and five organ failure scores [the cardiovascular, CNS, respiratory, renal, and coagulation scores]), as well as age and gender, to enter into the multivariate model (Cox proportional hazard model). In the final model, in which seven variables remained, ARDS development (hazard ratio [HR], 7.448; 95\% confidence interval [CI], 2.894 to 19.168), lactate (HR, 1.219; 95\% CI, 1.087 to 1.367 ), and the renal score (HR, 2.034; $95 \% \mathrm{CI}, 1.372$ to 3.015) were independently associated with the 28-day mortality. In the Kaplan-Meier survival curves for 28 day survival, the mean survival time was significantly shorter in patients with ARDS than in those without ARDS (Fig. 5).

\section{DISCUSSION}

We report several interesting findings. First, among nonpulmonary organ dysfunctions, CNS dysfunction and coagulopathy were significantly associated with ARDS development in patients with septic bacteremia. Second, ARDS development was significantly associated with an increase in 28-day mortality.

We used the new Sepsis-3 definition, which aims to render diagnoses consistent and to facilitate early sepsis recognition [9]. The previous definitions of sepsis (Sepsis-1 and Sepsis-2 of 1991 and 2001, respectively) have been found to be invalid $[13,14]$. In addition, the task force that developed Sepsis-3 emphasized life-threaten- 
Table 4. Multivariate analysis for risk factors of ARDS development

\begin{tabular}{|c|c|c|c|c|}
\hline & \multicolumn{2}{|c|}{ Unadjusted OR } & \multicolumn{2}{|c|}{ Adjusted OR } \\
\hline & OR & $95 \% \mathrm{CI}$ & OR & $95 \% \mathrm{CI}$ \\
\hline Age & 0.977 & $0.945^{-1.009}$ & 0.979 & $0.936-1.023$ \\
\hline Male sex & 0.955 & $0.379-2.406$ & 1.022 & $0.310-3 \cdot 371$ \\
\hline Pneumonia sepsis & 3.935 & $1.475-10.498$ & 4.590 & $1.177-17.895$ \\
\hline Gram-negative infection & 0.307 & $0.117-0.808$ & 0.377 & $0.107-1.326$ \\
\hline Coagulation score & 1.836 & $1,219-2,766$ & 2.669 & $1.438-4.954$ \\
\hline CNS score & 2.117 & $1.355-3.309$ & 1.917 & $1.097-3.348$ \\
\hline C-reactive protein & 1.006 & $1.001-1.011$ & 1.007 & $1.001-1.013$ \\
\hline
\end{tabular}

Hosmo-Lemeshow test, chi-sqaure $=3.875(\mathrm{df}=8), p=0.398$.

ARDS, acute respiratory distress syndrome; OR, odds ratio; CI, confidence interval; CNS, central nervous system.

Table 5. Comparisons between 28 -day survivors and nonsurvivors

\begin{tabular}{|c|c|c|c|c|}
\hline Variable & All patients $(n=125)$ & Survivors $(n=102)$ & Nonsurvivors $(\mathrm{n}=23)$ & $p$ value \\
\hline Age, yr & $73(59.0-80.0)$ & $73.0(59.0-79.0)$ & $75.0(59.0-81.0)$ & 0.954 \\
\hline Sex, male/female & $67 / 58$ & $53 / 49$ & $14 / 9$ & 0.439 \\
\hline \multicolumn{5}{|l|}{ Comorbid illness } \\
\hline Diabetes & $54(43.2)$ & $41(40.2)$ & $13(56 \cdot 5)$ & 0.153 \\
\hline Hypertension & $62(49.6)$ & $52(51.0)$ & $10(43 \cdot 5)$ & 0.516 \\
\hline COPD & $3(2.4)$ & $3(2.9)$ & 0 & 1.000 \\
\hline CKD & $21(16.8)$ & $14(13.7)$ & $7(30.4)$ & 0.066 \\
\hline Heart diseases & $18(14 \cdot 4)$ & $11(10.8)$ & $7(30.4)$ & 0.023 \\
\hline Cancer & $10(8.0)$ & $8(7.8)$ & $2(8.7)$ & 1.000 \\
\hline Pneumonia vs. nonpneumonia & $28 / 97$ & $19 / 83$ & $9 / 14$ & 0.033 \\
\hline Sepsis/septic shock & $64 / 61$ & $55 / 47$ & $9 / 14$ & 0.200 \\
\hline MDR pathogens & $43(34 \cdot 4)$ & $34(33 \cdot 3)$ & $9(39.1)$ & 0.597 \\
\hline Gram (+) vs. $(-)$ pathogens & $31 / 94$ & $20 / 82$ & $11 / 12$ & 0.005 \\
\hline Inappropriate antibiotics & $11(8.9)$ & $8(7 \cdot 9)$ & $3(13.0)$ & 0.426 \\
\hline Mechanical ventilation & $35(28.5)$ & $19(18.6)$ & $16(69.6)$ & $<0.001$ \\
\hline ARDS & $22(17.6)$ & $8(7.8)$ & $14(60.9)$ & $<0.001$ \\
\hline Vasopressors & $81(64.8)$ & $63(61.8)$ & $18(78.3)$ & 0.135 \\
\hline $\mathrm{PaO}_{2} / \mathrm{FiO}_{2}$ & $250.0(180.0-303.5)$ & $260(210.0-320.0)$ & $170.0(100.0-254.0)$ & 0.003 \\
\hline Lactate, mmol/L & $3.6(2.4-5.8)$ & $3.1(2.5-5 \cdot 3)$ & $5 \cdot 6(3.1-11.4)$ & 0.011 \\
\hline $\mathrm{BNP}, \mathrm{pg} / \mathrm{mL}$ & $297.4(138.5-973.4)$ & $273.9(128.4-780.3)$ & $1,040.4\left(234 \cdot 5^{-2,549 \cdot 4)}\right.$ & 0.005 \\
\hline Blood urea nitrogen & $31.1(20.4-55.0)$ & $28.9(19.1-53.4)$ & $42.1(32.4-78.2)$ & 0.041 \\
\hline C-reactive protein & $172.9(127.4-231.5)$ & $170.6(119.9-228.2)$ & $177.9(142.7-272.0)$ & 0.682 \\
\hline SOFA & $7.0(5 \cdot 5-10.0)$ & $7.0(5.0-9.0)$ & $13.0(9.0-14.0)$ & $<0.001$ \\
\hline SAPS II & $44.0(35.0-56.0)$ & $41.0(33.0-48.0)$ & $61.0(57.0-77.0)$ & $<0.001$ \\
\hline
\end{tabular}

Values are presented as median (interquartile range) or number (\%).

COPD, chronic obstructive pulmonary disease; CKD, chronic kidney disease; MDR, multi-drug resistance; ARDS, acute respiratory distress syndrome; $\mathrm{PaO}_{2} / \mathrm{FiO}_{2}$, ratio of arterial oxygen tension to inspired oxygen fraction; BNP, brain natriuretic peptide; SOFA, sequential organ failure assessment; SAPS, simplified acute physiology score. 


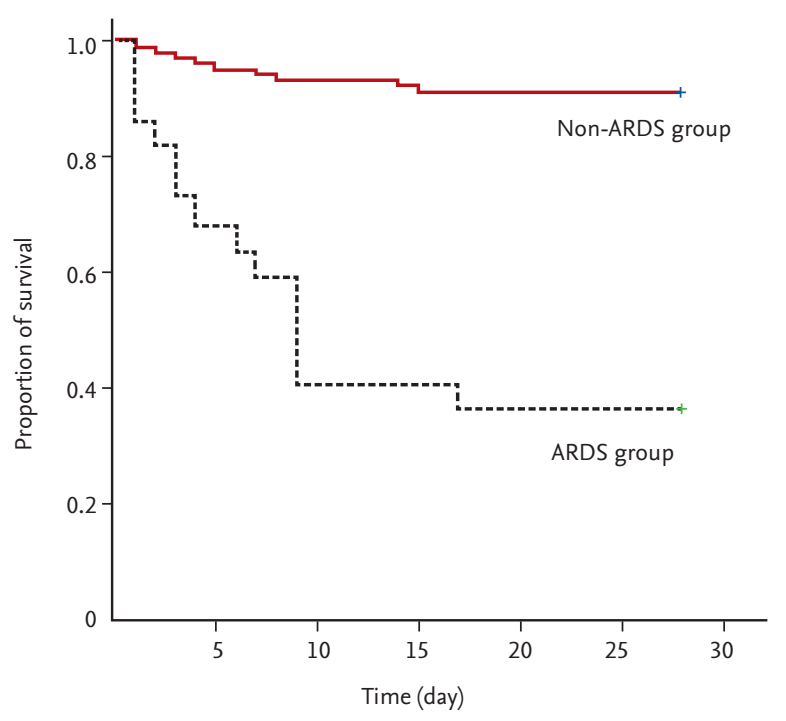

Figure 5. Kaplan-Meier survival curves of the 28-day survival of patients with and without acute respiratory distress syndrome (ARDS). The mean survival time was significantly shorter in patients with ARDS than in those without ARDS (13.9 \pm 2.4 days vs. $26.1 \pm 0.6$ days, log-rank test $p<0.001)$.

ing organ dysfunctions $[8,9,15]$. In this context, we evaluated the role played by nonpulmonary organ failure in ARDS development early in sepsis.

Sepsis is a common cause of ARDS. Although ARDS incidence varies by infection site, the risk factors for ARDS development remain unclear. Seethala et al. [2] found that the Acute Physiology and Chronic Health Evaluation (APACHE) II score, pneumonia, pancreatitis, an acute abdomen, and shock were independently predictive of ARDS in septic patients. Moss et al. [16] found that pneumonia was an independent predictor of ARDS, consistent with what was found by Gattinoni et al. [17], who reported differences in respiratory mechanics and responses to positive end-expiratory pressure depending on whether ARDS was caused by a pulmonary or nonpulmonary source. In this study, we did not calculate lung injury scores, but patients with pneumonia (thus with higher respiratory SOFA scores) exhibited a significantly higher incidence of ARDS and a trend toward higher mortality, compared with the nonpneumonia group. Therefore, pulmonary infection may be significant in terms of ARDS development and may be associated with poor in-hospital outcomes.

Activation of coagulation and an unbalanced inflammatory reaction are characteristic of both ARDS and sepsis, triggering fibrin deposition in capillary beds, in turn causing organ dysfunction [18]. Recently, both neutrophil extracellular trapping and platelet aggregation have been recognized as key players during ARDS development in septic patients $[19,20]$. We found that the coagulopathic score was associated with ARDS development and a higher score seemed to be associated with mortality ( $p=0.032$ ) (Fig. $3 \mathrm{~B}$ ).

We believe that our work re-emphasizes the important role played by activation of coagulation during ARDS development and for 28-day mortality. In addition, CNS dysfunction was associated with ARDS development. It may develop secondarily to hypoxia, hypoglycemia, and/or hypotension in patients with sepsis and may reflect sepsis-induced multiorgan dysfunction. Although relevant data are sparse, Moss et al. [16] found that patients who chronically abuse alcohol had higher CNS scores and were at a higher risk of ARDS. In this context, we suggest that the impact of sepsis-associated CNS dysfunction on patient outcomes be investigated further.

The incidence of ARDS in the present study (17.6\%) was lower than that in previous studies $[16,21]$, perhaps because of differences in the severity of illness. Fifty-one percent of our patients had sepsis (vs. septic shock, $49.0 \%$ ), and $37.6 \%$ had sepsis of urinary tract origin, which is regarded as less severe than other forms of sepsis. Of the patients evaluated by Seethala et al. [2], only $9.2 \%$ were in shock; the mean APACHE II score was 11.7, and the ARDS incidence was also low (6.2\%). However, Eggimann et al. [6] evaluated patients similar to ours and reported an ARDS incidence of $15.8 \%$, close to our figure. In the present study, ARDS development was significantly associated with an increased 28-day mortality, which contrasts previous observational studies $[6,22,23]$. After adjusting for the severity of illness and nonpulmonary organ dysfunction, Eggimann et al. [6] showed that, ARDS development was not independently associated with short-term mortality. This disparity may be the result of the different mortality rates of both ARDS and non-ARDS patients between the two studies. In particular, the 28-day and in-hospital mortality rates (63.6\% and $72.7 \%$, respectively) in our ARDS group seemed to be higher than those in previous studies. This could be attributable to their high severity of illness at the time of ICU admission (i.e., SAPS 
II score, 58 ; estimated death rate, $63 \%$ ), and the fact that ARDS occurred in patients with pre-existing septic bacteremia.

Our study had several limitations. First, our patient numbers were small, and the retrospective nature of the work may have introduced unidentified bias. Second, our study population was heterogeneous in terms of the origin of sepsis. In particular, the frequency of sepsis derived from urinary tract infections (usually associated with a good prognosis) was higher than that of previous studies. However, we enrolled only patients with septic bacteremia to ensure a homogeneous study population. We also investigated the appropriateness of empirical antibiotic therapy and pathogen multidrug resistance. To our knowledge, very few data are available on the role played by nonpulmonary organ dysfunction for ARDS development in patients with sepsis. Previous studies suggested that the site of infection does not independently affect mortality $[22,23]$. Hence, physicians need to pay more attention to organ dysfunction than to specific infection sites when identifying high-risk patients and initiating early interventions.

In conclusion, our data indicate that among nonpulmonary organ dysfunctions, CNS dysfunction and coagulopathy are independent risk factors for ARDS development in patients with septic bacteremia, and ARDS significantly increases 28-day mortality.

\section{KEY MESSAGE}

1. Among nonpulmonary organ dysfunctions, central nervous system dysfunction and coagulopathy were independent risk factors for the development of acute respiratory distress syndrome (ARDS) in patients with bacteremia sepsis.

2. The development of ARDS was significantly associated with an increase in 28-day mortality in patients with bacteremic sepsis.

\section{Conflict of interest}

No potential conflict of interest relevant to this article was reported.

\section{Acknowledgments}

The authors want to thank Young Soo Ju (Hallym University Sacred Heart Hospital) for his contribution to statistical analysis.

\section{REFERENCES}

1. Torio CM, Andrews RM. National inpatient hospital costs: the most expensive conditions by payer, 2011. Statistical Brief \#160. In: United States. Agency for Healthcare Research and Quality. Healthcare Cost and Utilization Project (HCUP) Statistical Briefs. Rockville (MD): Agency for Healthcare Research and Quality (US), 2006 [cited 2018 May 29]. Available from: https://www.ncbi.nlm. nih.gov/books/NBK169005/.

2. Seethala RR, Hou PC, Aisiku IP, et al. Early risk factors and the role of fluid administration in developing acute respiratory distress syndrome in septic patients. Ann Intensive Care 2017;7:11.

3. Ferguson ND, Frutos-Vivar F, Esteban A, et al. Clinical risk conditions for acute lung injury in the intensive care unit and hospital ward: a prospective observational study. Crit Care 2007;11:R96.

4. Del Sorbo L, Slutsky AS. Acute respiratory distress syndrome and multiple organ failure. Curr Opin Crit Care 2011;17:1-6.

5. Russell JA, Singer J, Bernard GR, et al. Changing pattern of organ dysfunction in early human sepsis is related to mortality. Crit Care Med 2000;28:3405-3411.

6. Eggimann P, Harbarth S, Ricou B, et al. Acute respiratory distress syndrome after bacteremic sepsis does not increase mortality. Am J Respir Crit Care Med 2003;167:12101214 .

7. Mikkelsen ME, Shah CV, Meyer NJ, et al. The epidemiology of acute respiratory distress syndrome in patients presenting to the emergency department with severe sepsis. Shock 2013;40:375-381.

8. Shankar-Hari M, Phillips GS, Levy ML, et al. Developing a new definition and assessing new clinical criteria for septic shock: for the third international consensus definitions for sepsis and septic shock (Sepsis-3). JAMA 2016;315:775-787.

9. Singer M, Deutschman CS, Seymour CW, et al. The third international consensus definitions for sepsis and septic shock (Sepsis-3). JAMA 2016;315:801-810. 
10. Dellinger RP, Levy MM, Rhodes A, et al. Surviving sepsis campaign: international guidelines for management of severe sepsis and septic shock: 2012. Crit Care Med 2013;41:580-637.

11. Dellinger RP, Levy MM, Carlet JM, et al. Surviving sepsis campaign: international guidelines for management of severe sepsis and septic shock: 2008. Intensive Care Med 2008;34:17-6o.

12. ARDS Definition Task Force, Ranieri VM, Rubenfeld GD, et al. Acute respiratory distress syndrome: the Berlin Definition. JAMA 2012;307:2526-2533.

13. American College of Chest Physicians/Society of Critical Care Medicine Consensus Conference: definitions for sepsis and organ failure and guidelines for the use of innovative therapies in sepsis. Crit Care Med 1992;20:864874.

14. Levy MM, Fink MP, Marshall JC, et al. 2001 SCCM/ESICM/ACCP/ATS/SIS international sepsis definitions conference. Intensive Care Med 2003;29:530-538.

15. Seymour CW, Liu VX, Iwashyna TJ, et al. Assessment of clinical criteria for sepsis: for the third international consensus definitions for sepsis and septic shock (Sepsis-3). JAMA 2016;315:762-774.

16. Moss M, Parsons PE, Steinberg KP, et al. Chronic alcohol abuse is associated with an increased incidence of acute respiratory distress syndrome and severity of multiple organ dysfunction in patients with septic shock. Crit Care Med 2003;31:869-877.

17. Gattinoni L, Pelosi P, Suter PM, Pedoto A, Vercesi P, Lissoni A. Acute respiratory distress syndrome caused by pulmonary and extrapulmonary disease. Different syndromes? Am J Respir Crit Care Med 1998;158:3-11.

18. Frantzeskaki F, Armaganidis A, Orfanos SE. Immunothrombosis in acute respiratory distress syndrome: cross talks between inflammation and coagulation. Respiration 2017;93:212-225.

19. Miyashita T, Ahmed AK, Nakanuma S, et al. A three-phase approach for the early identification of acute lung injury induced by severe sepsis. In Vivo 2016;30:341-349.

20. Xue M, Sun Z, Shao M, et al. Diagnostic and prognostic utility of tissue factor for severe sepsis and sepsis-induced acute lung injury. J Transl Med 2015;13:172.

21. Moss M, Bucher B, Moore FA, Moore EE, Parsons PE. The role of chronic alcohol abuse in the development of acute respiratory distress syndrome in adults. JAMA 1996;275:50-54.

22. Sevransky JE, Martin GS, Mendez-Tellez P, et al. Pulmonary vs nonpulmonary sepsis and mortality in acute lung injury. Chest 2008;134:534-538.

23. Sheu CC, Gong MN, Zhai R, et al. The influence of infection sites on development and mortality of ARDS. Intensive Care Med 2010;36:963-970. 